\title{
Beam-Induced Radiation in a Muon Storage Ring for a Neutrino Factory *
}

\author{
N.V. Mokhov and C.J. Johnstone \\ Fermilab, P.O. Box 500, Batavia, IL 60510, USA ${ }^{1}$
}

\begin{abstract}
Beam-induced radiation effects have been simulated for a $30-\mathrm{GeV}$ and a $50-\mathrm{GeV}$ muon storage ring designed for a neutrino factory. Calculations include power dissipation and residual dose rates on lattice components. It is shown that by appropriately shielding the superconducting magnets quench stability can be achieved along with residual dose rates which allow hands-on maintenance. Studies on tunnel shielding futher showed that unlimited-occupancy areas could be achieved during machine operation if such enclosures are placed to the inside of the ring. Finally, neutrino-induced radiation levels were computed to large distances for outside the arcs and downstream of the production straights.
\end{abstract}

Key words: Neutrino factory, muon storage ring, beam loss, energy deposition, superconducting magnets, radiation environment

PACS: 13.35.Bv, 41.60.Ap, 85.25.Ly, 87.53.Wz

\section{Introduction}

The energy deposition from beam loss and muon decay has been simulated for both a $30-\mathrm{GeV}[1]$ and a $50-\mathrm{GeV}$ [2] muon storage ring using the Monte Carlo code, MARS [3,4]. Except in energy, both rings are similar: a racetrack geometry with long, normal-conducting magnets in production straights and short, superconducting (SC) ones in the arcs. These magnets were profiled in detail in the simulations. From these calculations, prompt and residual radiation levels have been determined in the lattice magnets, around the tunnel and at large distances from the complex.

\footnotetext{
* Work supported by the Universities Research Association, Inc., under contract DE-AC02-76CH00300 with the U. S. Department of Energy.

1 E-mail: mokhov@fnal.gov
} 


\section{Dynamic Losses in Ring}

Lattice functions for the arcs and production regions are shown in Fig. 1. Both rings have arcs composed of FODO cells, $9.8 \mathrm{~m}$ in length, which use 1-m long quadrupoles of strength $45 \mathrm{~T} / \mathrm{m}$ and 2.4-m long dipoles with a $6 \mathrm{~T}$ field. Views of the magnet as modeled in MARS are shown in Fig. 2.
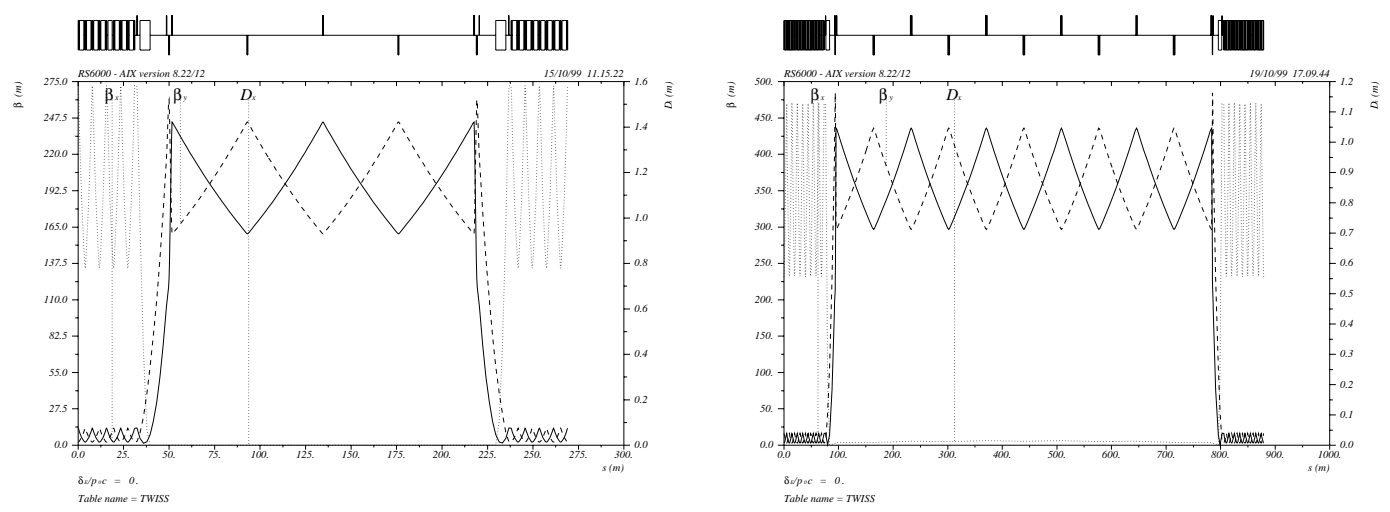

Fig. 1. Lattice functions for the $30 \mathrm{GeV}$ (left) and $50 \mathrm{GeV}$ (right) half rings including the downward production straights.
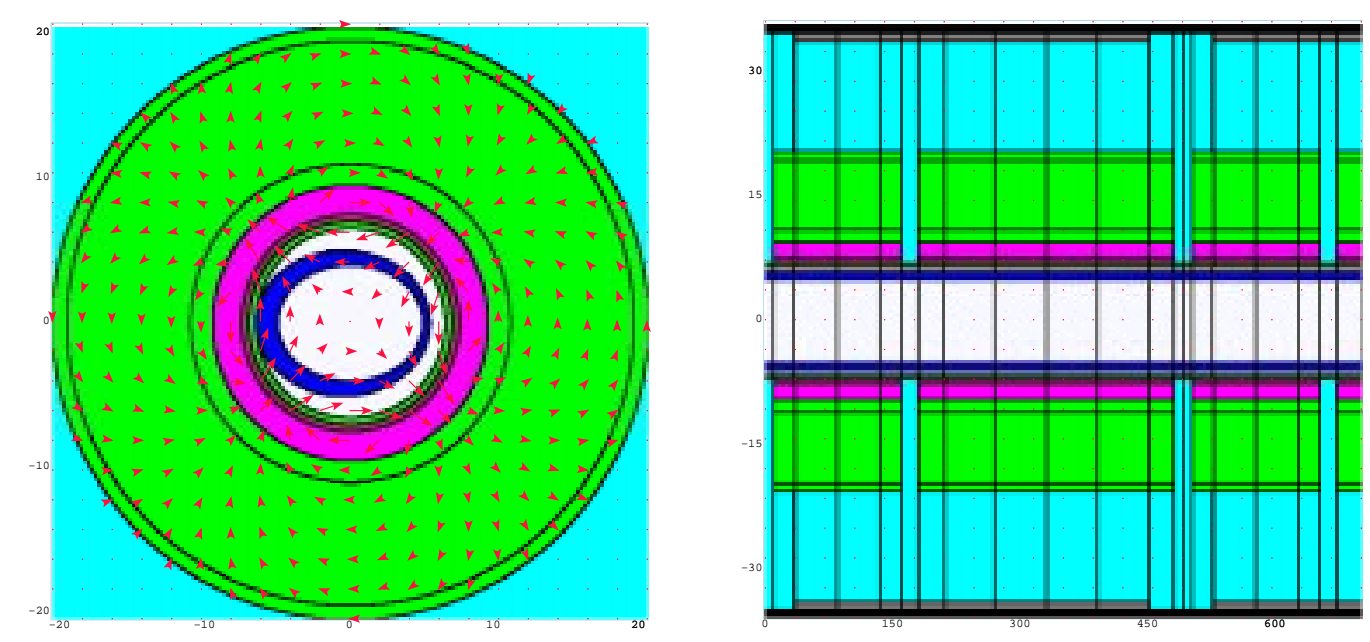

$\stackrel{x}{h}$

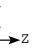

Fig. 2. MARS arc model: dipole cross-section with an eccentric bore tube (left) and a longitudinal view of the arc cells (right).

At $50 \mathrm{GeV}$, the muon decay rate is $2 \cdot 10^{12} \times 15 \times 165 \times 3.21 \cdot 10^{-6}=1.6 \cdot 10^{10}$ decays $/ \mathrm{m} / \mathrm{s}$. The beam's spatial and angular distributions are simulated using a rms transverse emittance of $3500 \pi \mathrm{mm}-\mathrm{mrad}$ and a rms $\Delta p / p$ of $1 \%$. Muon decay, $\mu \rightarrow e \nu \tilde{\nu}$, is characterized in the code using the exact decay kinematics. The decay electrons and, also, the synchrotron photons subsequently emitted by these electrons, eventually travel outside the machine aperture and induce electromagnetic showers which then propagate through the lattice elements. 
These showers are fully simulated with MARS14 [4] so that the corresponding heat load deposited in the magnets can be calculated along with the radiation fields generated in the tunnel and surrounding areas. Details of the power density distribution in the SC magnet components are essential to determining the quench stability of the magnets and the potential impact on the machine operation. The corresponding heat load delivered to the magnets also specifies the cooling requirements for the ring, both in water and cryogenic capability. Calculation of the radiation fields, during machine operation and residual activation, has been converted to equivalent dose rates, allowing occupancy of nearby enclosures and maintenance issues to be resolved.

\section{Arc Magnets}

For a $240-\mathrm{kW}$ circulating muon beam and a $1750-\mathrm{m}$ circumference, $84 \mathrm{~kW}$ of power is dissipated in the ring at $50 \mathrm{GeV}$, or 47.8 Watts per meter on average. Calculations show that most of this power must be intercepted by a thick tungsten tube inserted in the SC magnet bore. Longitudinal distribution of both power density and power dissipation oscillate periodically along the length of the arc cell. From Fig. 3(left), which is the case with bent dipoles (dipoles with a sagitta) and a 1-cm thick tungsten bore tube, it can be seen that $90 \%$ of the power is dissipated in the protective bore tube and only about $10 \%$ of power is dissipated in the SC coils. The power density peaks in the orbit plane exhibiting a strong azimuthal variation as shown in Fig. 3(right) for the case with bent dipoles and an eccentric tungsten bore tube.
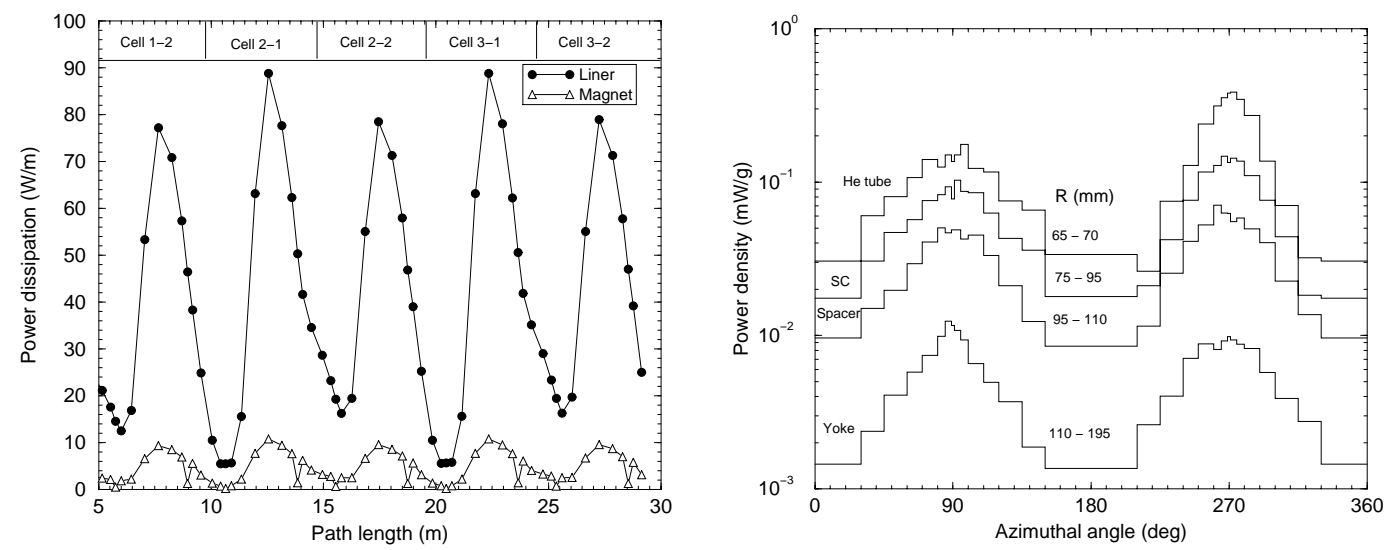

Fig. 3. Power dissipation along the arc lattice magnets (left) and azimuthal distribution of power density in the dipole components at the longitudinal peak (right).

Using MARS, thorough optimization of the liner has been performed to study its effectiveness in reducing the power dissipated in the SC magnet coils and body. Table 1 compares the effect of a bore tube made of different materials. Due to the electromagnetic origin of energy deposition, the heavier material, 
the stronger the interaction, and the more localized the showers - hence the increase in protection with atomic number. With a tungsten liner, the induced activation and therefore the residual dose rates on the magnets become acceptable for hands-on maintenance (Table 2).

Peak power density in SC coils is noticeably lower in a bent dipole compared to a straight one (Table 3). Furthermore, since decay electrons are swept to the inward side of the dipole, thickening that side of the bore tube is particularly effective in reducing the power dissipated in the magnets (Fig. 2(left)). The combination of a bent dipole and an eccentric tungsten bore reduces peak power density in the SC coils (quench stability) and dynamic heat load to the arc magnets (cryogenic heat capacity) to the tolerable levels of $P_{\max } \leq 1 \mathrm{~mW} / \mathrm{g}$ and $\mathcal{P}_{\text {max }} \leq 10 \mathrm{~W} / \mathrm{m}$, respectively (Table 3 ). An eccentric tungsten shield can be made of a 1-mm thick stainless steel pipe $(100 \times 80 \mathrm{~mm}$ elliptical aperture $)$ and a 1-mm thick stainless steel pipe $(122 \times 102 \mathrm{~mm}$ elliptical aperture $)$ shifted inward by $5 \mathrm{~mm}$ with a space between filled with a tungsten-like material. With such a tube, the peak power density in the coil is only $0.15 \mathrm{~mW} / \mathrm{g}$, with the peak power dissipation in the bore tube (nitrogen temperature) and the rest of the magnet (helium temperature) about 90 and $9 \mathrm{~W} / \mathrm{m}$, respectively. Averaged over the arc length the values are approximately a factor of two lower than the peak.

Table 1

Power dissipation $(\mathrm{W} / \mathrm{m})$ averaged over $50-\mathrm{GeV}$ arc magnet components with a 1cm thick liner made of tungsten, copper and aluminum: liner $(100 \times 80 \mathrm{~mm}$ ID $)$, SS He tube $(65<\mathrm{r}<70 \mathrm{~mm})$, SC coil $(75<\mathrm{r}<95 \mathrm{~mm})$, rest of the magnet $(\mathrm{r}>95 \mathrm{~mm})$ and sum in the magnet $(\mathrm{r}>65 \mathrm{~mm})$.

\begin{tabular}{|c|c|c|c|c|c|}
\hline Material & Liner & Tube & Coil & Rest & Sum at $\mathrm{r}>65 \mathrm{~mm}$ \\
\hline $\mathrm{W}$ & 42.75 & 1.09 & 1.46 & 2.53 & 5.08 \\
$\mathrm{Cu}$ & 29.88 & 4.83 & 5.37 & 6.36 & 16.56 \\
$\mathrm{Al}$ & 5.25 & 11.63 & 15.08 & 13.94 & 40.65 \\
\hline
\end{tabular}

Table 2

Longitudinal maximum of residual dose rate at contact $(\mu \mathrm{Sv} / \mathrm{hr})$ after 30 day irradiation and 1 day cooling with a $1-\mathrm{cm}$ thick liner made of three materials for a $50-\mathrm{GeV}$ arc.

\begin{tabular}{|c|c|c|c|c|}
\hline Material & Liner & SC coil & He vessel & Outer vessel \\
\hline $\mathrm{W}$ & 13.1 & 6.4 & 5.0 & 3.2 \\
$\mathrm{Cu}$ & 80.5 & 12.3 & 9.1 & 4.8 \\
$\mathrm{Al}$ & 187 & 32.0 & 15.0 & 5.6 \\
\hline
\end{tabular}


Table 3

Peak power density $P_{\max }(\mathrm{mW} / \mathrm{g})$ in $\mathrm{SC}$ coils and peak power dissipation $\mathcal{P}_{\max }$ $(\mathrm{W} / \mathrm{m})$ in a bore tube and in the magnet $(\mathrm{r}>65 \mathrm{~mm})$ for a $50-\mathrm{GeV}$ arc.

\begin{tabular}{|l|l|c|c|c|}
\hline Dipole & Bore tube & $P_{\max }(\mathrm{mW} / \mathrm{g})$ & $\mathcal{P}_{\max }(\mathrm{W} / \mathrm{m})$ & $\mathcal{P}_{\max }(\mathrm{W} / \mathrm{m})$ \\
\hline & & SC coil & Bore tube & Magnet \\
\hline Bent & $1-\mathrm{cm} \mathrm{W}$ & 0.285 & 88.75 & 10.84 \\
Bent & $1.5 x 0.5 \mathrm{~W}$ & 0.147 & 90.45 & 8.875 \\
Straight & $1-\mathrm{cm} \mathrm{W}$ & 0.424 & 109.7 & 13.87 \\
Bent & $1.5 x 0.5 \mathrm{~Pb}$ & 0.352 & 81.92 & 16.68 \\
Bent & $1-\mathrm{cm} \mathrm{Cu}$ & 2.790 & 54.61 & 40.11 \\
\hline
\end{tabular}

\section{Radiation In and Around Tunnel}

It is found that the normal occupancy limit of $0.25 \mathrm{mR} / \mathrm{hr}$ is met with $2 \mathrm{~m}$ of dolomite-type shielding below, above, and radially inward from the arc tunnel enclosure walls. Six meters of such a shielding is needed to meet this limit radially outward from the ring [4] (Fig. 4(left)). Conveniently, power supply rooms and other underground enclosures should be placed on the inward side of the arc tunnel permitting unlimited occupancy even during machine operation.
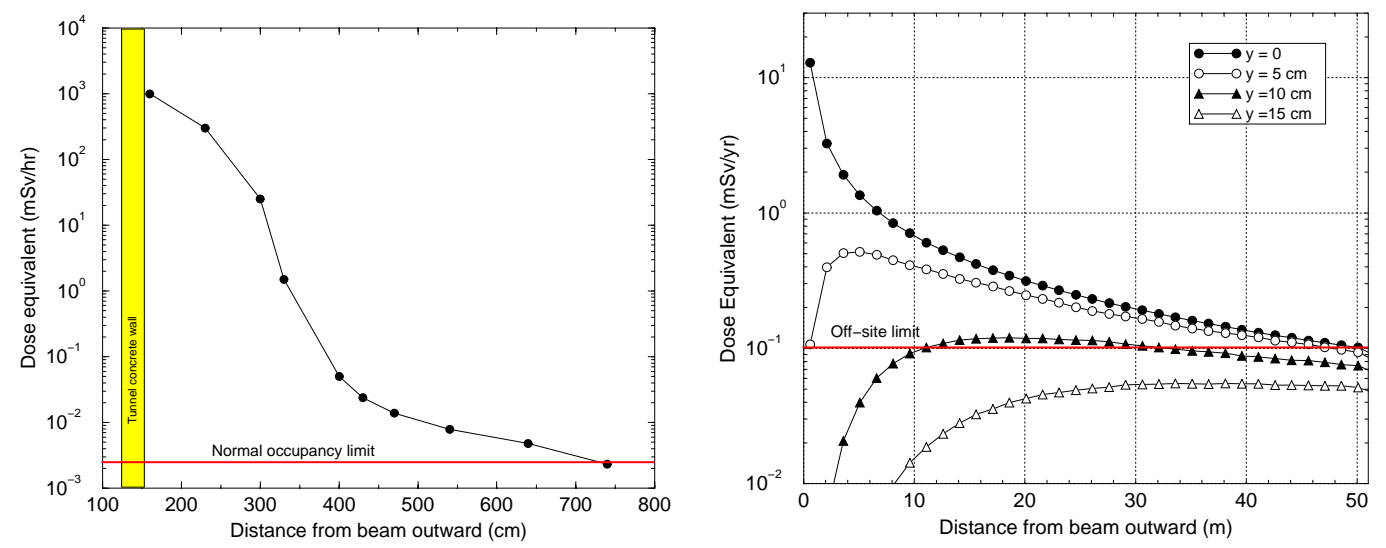

Fig. 4. Non-neutrino dose rate radially outward from the arc tunnel enclosure in a $50 \mathrm{GeV}$ muon storage ring (left) and neutrino-induced annual maximum dose equivalent in a phantom embedded in soil outward from a $50 \mathrm{GeV}$ arc tunnel at several heights with respect to the orbit plane (right).

In MARS14 calculations, the decay neutrinos are propagated in the code through the dirt surrounding the tunnel, where they interact, and create daughter showers which produce radiation at very large distances [5]. The off-site limit of $10 \mathrm{mR} / \mathrm{yr}$ or $0.1 \mathrm{mSv} / \mathrm{yr}$ is reached $50 \mathrm{~m}$ radially outward from the beam's orbit (Fig. 4(right)). However, the height of the radiation disk reaches only $\pm 10 \mathrm{~cm}$ above and below the orbit plane. Neutrino-induced radiation downstream of the straight sections is more severe than from the arcs. The off-site 
limit of is only met after $4.2 \mathrm{~km}$ and $1.8 \mathrm{~km}$ for $50 \mathrm{GeV}$ and $30 \mathrm{GeV}$ muon storage ring, respectively [5]. The maximum half-width of an off-site limit isocontour is $4.3 \mathrm{~m}$ and $2.7 \mathrm{~m}$, respectively.

\section{Conclusions}

The radiation load to the $\mathrm{SC}$ coils of the arc magnets due to $50 \mathrm{GeV}$ muon beam decays is reduced to the levels acceptable for quench stability by a tungsten (or even lead) eccentric bore tube. With the optimal bore tube, the peak power density in the coil is $0.15 \mathrm{~mW} / \mathrm{g}$ and the peak power dissipation in the bore tube (nitrogen temperature) and in the rest of the magnet (helium temperature) is about 90 and $9 \mathrm{~W} / \mathrm{m}$, respectively. Averaged over the arc length, the power dissipation values are approximately a factor of two lower than the peak. Residual dose rates on the magnets also fall below the regulated limits for hands-on maintenance. Normal occupancy limit is achieved by providing 2 meters of dolomite type shielding below, above and radially inward from the arc tunnel enclosure walls. Radially outward from the beam orbit conventional radiation dictates six meters of such shielding, while an equivalent reduction of the neutrino-induced radiation requires 50 meters. Power supply rooms and other underground enclosures obviously should be placed on the inward side of the arc tunnel. The off-site limit downstream of the straight sections is satisfied only at a distance of $4.2 \mathrm{~km}$ for a $50 \mathrm{GeV}$ complex.

\section{References}

[1] C.J. Johnstone, Large Acceptance 30-GeV Muon Storage Ring for Neutrino Production: Lattice Design, to be published in: Proc. of NuFact'99 Workshop (Lyon, France, July 5-9, 1999).

[2] C.J. Johnstone, in: N. Holtkamp and D. Finley, eds., A Feasibility Study of a Neutrino Source Based on a Muon Storage Ring, Fermilab-Pub-00/108-E (2000).

[3] N.V. Mokhov, The MARS Code System User's Guide, Fermilab-FN-628 (1995); N.V. Mokhov et al., MARS Code Developments, Fermilab-Conf-98/379 (1998); http://www-ap.fnal.gov/MARS/

[4] N.V. Mokhov, MARS Code Developments, Benchmarking and Applications, in: Proc. of ICRS-9 International Conference on Radiation Shielding (Tsukuba, Ibaraki, Japan, 1999), J. Nucl. Sci. Tech. 1 (2000) 167-171; Fermilab-Conf00/066 (2000).

[5] N.V. Mokhov and A. Van Ginneken, Neutrino Radiation at Muon Colliders and Storage Rings, ibid, pp. 172-179; Fermilab-Conf-00/065 (2000). 